\title{
SÍNDROME DE WORSTER-DROUGHT
}

\section{Relato de caso e distinção em relação à síndrome de Foix-Chavany-Marie}

\author{
Fernanda Queirós', Gleison Duarte'2, Cecília Correia³, \\ Juliana Gomes Sérgio", Camila Vila-Nova5, Rita Lucena ${ }^{6}$
}

\begin{abstract}
RESUMO - A síndrome de Worster-Drought é entidade clínica decorrente de lesão do trato cortico-nuclear. Não existe etiologia definida e caracteriza-se por alteração do controle voluntário e involuntário dos músculos que movimentam lábios, mandíbula, língua, pálato mole e faringe. As principais manifestações clínicas são alterações na fonação e deglutição. Descrevemos o caso de paciente do sexo feminino, 15 anos, que apresenta a referida síndrome. Os achados clínicos e radiológicos são comparados com os dados descritos na literatura. Questiona-se a ligação entre síndrome de Worster-Drought e a de Foix-ChavanyMarie, bem como a possível relação entre o uso de drogas abortivas e transtorno oromotor semelhante ao identificado nessas enfermidades.
\end{abstract}

PALAVRAS-CHAVE: síndrome de Worster-Drought, síndrome de Foix-Chavany-Marie, alterações fonoaudiológicas, abortivos, motricidade oral, alterações cognitivas.

\begin{abstract}
Worster-Drought syndrome: case report and distinction in relation to Foix-Chavany-Marie syndrome
ABSTRACT - Worster-Drought syndrome is a clinical entity resulting from cortico-nuclear tract or cerebral cortex injury, without defined etiology, characterized by alteration of the muscles that control the lips, jaw, tongue, soft palate and pharynx. The main clinical manifestations are alterations in the fonation and deglutition. We describe the case of a 15 year old female patient, displaying the cited syndrome. The clinical, radiological and neurophysiological findings are compared with the described data in the literature. We question the connection between Worster-Drought and Foix-Chavany-Marie syndroms, as well as the possible relationship with use of abortive drugs and similar oromotor problems as found in these two syndromes.
\end{abstract}

KEY WORDS: Worster-Drought syndrome, speech pathology, Foix-Chavany-Marie syndrome, abortives, oral motricity, cognitive alterations.

A síndrome de Worster-Drought (SWD) foi descrita inicialmente pelo autor, em 1953, como paresia suprabulbar congênita, contrapondo-se ao termo "paresia pseudobulbar" usado para definir fraqueza ou paralisia da musculatura faciolinguofaringomastigatória, em que não existem evidências de comprometimento do neurônio motor inferior ${ }^{1}$. No artigo original, o autor descreveu que esta síndrome é provocada por lesão do trato cortico-nuclear, secundária a agenesia ou disgenesia - que pode ser provocada por uso de abortivos, como o misoprostol - apresentando variações do espectro clínico anteriormente descrito ${ }^{1}$. Por outro lado, a síndrome de Foix-Chavany-Marie (SFCM), descrita inicialmente em 1926, produz também prejuízo da mesma musculatura, mas com preservação das funções reflexas/automáticas. Esta síndrome é secundária a lesão opercular bilateral, possivelmente por defeito no desenvolvimento ou neuromigração cortical, produzindo o que se costumou denominar "síndrome opercular" ou síndrome perisylviana bilateral congênita ${ }^{2,3}$. A paresia suprabulbar congênita, ou Síndrome de Worter-Drought, é lesão do neurônio motor superior, que produz paresia envolvendo predominantemente a musculatura suprida pelos nervos vago (X craniano), hipoglosso

Universidade Federal da Bahia, Salvador BA, Brasil (UFBA): ${ }^{1}$ Fonoaudióloga clínica, mestranda em Neurociências (UFBA); ${ }^{2}$ Acadêmico de Medicina (UFBA); ${ }^{3}$ Médica neurofisiologista (Hospital São Rafael, Salvador - BA, Brasil); ${ }^{4}$ ssicóloga, especialista em linguagem; ${ }^{5}$ Fonoaudióloga clínica, aluna especial do mestrado em Neurociências (UFBA); ${ }^{6}$ Neuropediatra, docente da UFBA, Doutora em Neurociências.

Recebido 5 Dezembro 2003, recebido na forma final 3 Maio 2004. Aceito 4 Junho 2004.

Dr. Fernanda Costa de Queirós - Rua Boa Fé 72E - 41185-290 Salvador BA - Brasil. E-mail: nandafono@terra.com.br 
(XII craniano) e, algumas vezes, pelo VII neuro (nervo facial), sendo considerada por alguns autores como uma forma de paralisia cerebral ${ }^{2,4}$. Clinicamente, caracteriza-se por espasticidade e fraqueza da língua, palato mole e lábios, o que produz desordens lingüísticas e da deglutição como sintomas predominantes. Em casos graves, pode ocorrer também fraqueza na musculatura laríngea e faríngea. Associada a espasticidade, a fraqueza ainda prejudica os movimentos laterais e de protusão da língua, mas não produz atrofia ou fasciculações'.

Inicialmente, os indivíduos apresentam dificuldade de sucção, seguida de atraso na fala. A disartria se caracteriza por hipernasalidade, dificuldade na produção de consoantes linguais (" $t$ ", " $d$ ", " $n$ ", "I") e labiais ("m", "b", "p"), além de movimentos velares limitados. Nos casos mais graves, pode haver anartria ${ }^{1}$. Caracteristicamente, ocorrem vigorosos espasmos reflexos mandibulares e escoamento de saliva, em conseqüência da redução da deglutição espontânea ${ }^{2,3}$. A mandíbula pode ter alterações morfológicas, além de falhas de alinhamento dos dentes. Como resultado dos distúrbios na deglutição associados à disfunção na tuba auditiva, também são muito freqüentes as infecções de orelha média, podendo provocar surdez condutiva. Em casos raros, têm sido descritas manifestações de espectro do autismo. Epilepsia ocorre em uma minoria, devendo ser tratada da forma convencional.

Relatamos e discutimos um caso de SWD.

\section{CASO}

Paciente do sexo feminino, 15 anos, portadora de paralisia cerebral mista, procurou atendimento fonoaudiológico devido a incapacidade de produzir fala e a dificuldade na alimentação. A genitora não teve acompanhamento pré-natal e fez uso de misoprostol, por via oral, no primeiro mês e prostigmine via intramuscular no segundo mês. Após trabalho de parto prolongado, foi realizada cesárea ( 24 horas após a rotura da bolsa). A criança nasceu com baixo peso $(2200 \mathrm{~g})$ e não chorou ao nascer. Não apresentou dificuldade aparente de sucção ao nascer ou nos meses seguintes. Sentou sem apoio com 18 meses e adquiriu marcha sem apoio aos 3 anos. O diagnóstico de paralisia cerebral foi definido com 1 ano e 1 mês.

O exame neurológico evidenciou defasagem cognitiva leve associada à hemiparesia espástica à direita e hemidistonia ipsolateral. A avaliação fonoaudiológica detectou dificuldades em relação à alimentação, causada por ausência de movimentos laterais e de protusão de língua, o que produzia acúmulo de alimentos no vestíbulo. A mastigação encontrava-se alterada, apresentando um padrão de amassamento, com reduzida movimen-

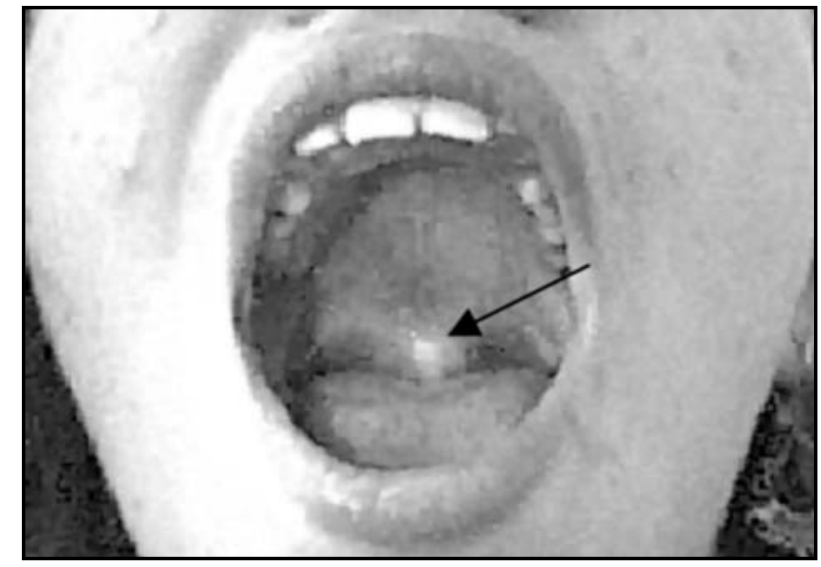

Fig 1. Emissão da vogal /al. Observar o rebaixamento do palato mole.

tação antero-posterior de língua. A paciente colocava alimentos sempre do lado esquerdo, sendo observada contração maior do músculo bucinador deste lado. Havia acentuada limitação da motricidade do orbicular dos lábios, determinando escoamento de líquido. Não usava canudo, não assoprava e nem conseguia inflar as bochechas. Apresentava escoamento de saliva constante e episódios de engasgos. Não apresentava reflexo de vômito. O palato mole tinha pouca movimentação, observando-se rebaixamento do mesmo na emissão da vogal oral /a/ (Fig 1) e a voz apresentava ressonância hipernasal. A comunicação era gestual. Não estava freqüentando escola, mas recebia orientação educacional no domicílio. O nível de aprendizagem correspondia ao de alfabetização. Compreendia ordens simples, mas mostrou dificuldade na compreensão de ordens complexas.

A ressonância magnética revelou diminutos focos de gliose no hemisfério cerebral direito, além de área de gliose maior $(2,0 \mathrm{~cm})$ no lobo temporal esquerdo, discreto espessamento bilateral dos giros corticais frontais, temporais superiores e insulares sugestivo de displasia cortical não lisencefálica.

A avalição eletroneuromiográfica, realizada no aparelho modelo Cantata/Dantec, 2 canais, mostrou potenciais compostos motores normais do nervo hipoglosso à direita e à esquerda, bem como potenciais de unidade motora de morfologia normal, padrão de interferência completo e recrutamento reduzido, sugerindo comprometimento do sistema nervoso central (Fig 2).

A avaliação cognitiva realizada incluiu uma combinação de técnicas e atividades pedagógicas com o objetivo de analisar os indicadores cognitivos presentes através da análise das respostas, dos pré-requisitos e das habilidades exigidas, distinguindo-se dos testes psicométricos que avaliam apenas o repertório já adquirido. Nesta proposta, foram aplicadas as provas do diagnóstico operatório ${ }^{5}$ e o Bender Infantil ${ }^{6}$, utilizado para definir índices de maturação percepto-motora. As provas do diagnóstico operatório aplicadas foram as de conser- 


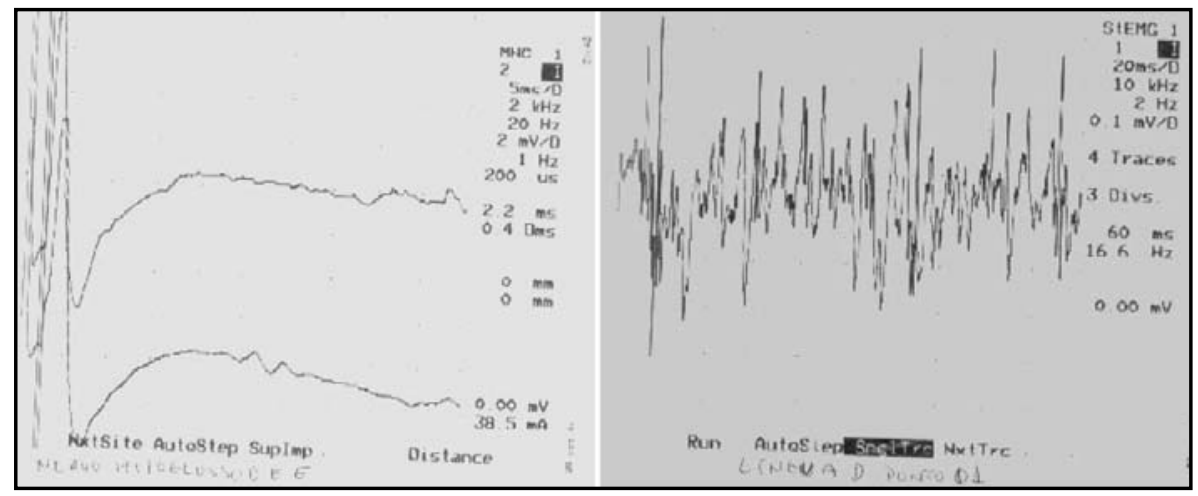

Fig 2. Achados da eletroneuromiografia. À esquerda composto motor do nervo hipoglosso direito e esquerdo. À direita Eletromiograma da língua à direita.

vação, classificação, seriação e combinação. Essas provas foram escolhidas já que não fazem exigência verbal em suas respostas. Funções cognitivas deficientes e incoordenação visomotora dificultaram a realização das provas. A paciente apresentou grande dificuldade na capacidade de compreensão verbal, necessitando de maior tempo na realização das atividades. Seu desempenho foi pior de acordo com o nível de abstração e com a maior exigência na organização viso-espacial. Ao Bender, nas provas denominadas de execução, que visam analisar a percepção, análise, síntese e reprodução de figuras abstratas, foi observada maior dificuldade, não conseguindo a reprodução das figuras a partir do momento em que o nível de complexidade era aumentado. As tarefas requerendo o reconhecimento e o domínio das dimensões, cor e tamanho não foram resolvidas quando se exigia a comparação e seriação entre essas dimensões. Apresentou resposta satisfatória apenas quando era solicitada a discriminação entre suas características. A performance nas provas de seriação, classificação e conservação manteve-se aquém do esperado. A resolução correta dessas tarefas restringiu-se aos níveis primários de seus conceitos. Reconheceu a escrita de letras e palavras e identificou números de até dois algarismos.

A genitora recebeu esclarecimentos sobre o estudo que seria realizado, concordando e assinando termo de Consentimento Informado.

\section{DISCUSSÃO}

Muitos estudos têm procurado estabelecer uma caracterização clínica que nos permitam distinguir as síndromes de Worster Drought e de Foix-Chavany-Marie. Adotando o conceito tradicional de distúrbios da linguagem como comprometimento da capacidade de produzir e compreender enunciados gramaticalmente estruturados e/ou semanticamente apropriados ${ }^{7}$, podemos concordar com a afirmação de Maw-Yuan et al. e de Weller et al. de que ambas as síndromes são um distúrbio da fala e não da linguagem ${ }^{3,8}$. Entre os distúrbios da fala, caracterizados por alteração da articulação, estão as disartrias e as dispraxias. Encontra-se freqüentemente na literatura a confusão entre estes dois termos, sendo os mesmos utilizados como sinônimos, quando na verdade não o são. Entende-se por disartria a "articulação imperfeita" da fala causada por uma lesão no sistema nervoso central ou periférico, caracterizada por fraqueza, incoordenação ou paralisia do aparelho fonador ${ }^{9}$. Por outro lado, o termo dispraxia refere-se uma desordem da articulação, que resulta da perda, causada por uma lesão cerebral, da capacidade de organizar o posicionamento da musculatura da fala e de seqüenciar os movimentos na produção (semi) espontânea de fonemas ou de uma seqüência de fonemas; porém esta dificuldade não é acompanhada por fraqueza ou lentidão significantes, ou incoordenação destes músculos nos movimentos reflexos ou automáticos ${ }^{9}$. Na SFCM, ocorre dispraxia bucofacial associada a disfasia motora e prejuízo da prosódia. Além disso, diferentemente do que foi identificado no caso descrito, na dispraxia não há alterações ligadas à sucção, mastigação e deglutição ${ }^{10}$. Assim, a paciente apresenta um quadro de disartria.

A SWD tem características distintas que nos permitem distinguí-la da SFCM. Nela, há comprometimento do córtex motor primário ou do trato córtico-nuclear, levando a uma síndrome parética que determina disartria. No entanto, alguns consideram as duas entidades como um contínuo de uma mesma doença, usando indistintamente as duas terminologias para descrever qualquer indivíduo com dispraxia ou disartria de origem congênita ou adquirida. Segundo Nevo et al. ${ }^{4}$ em relato de caso, achados clínicos e radiológicos semeIhantes demonstram haver uma continuidade entre 


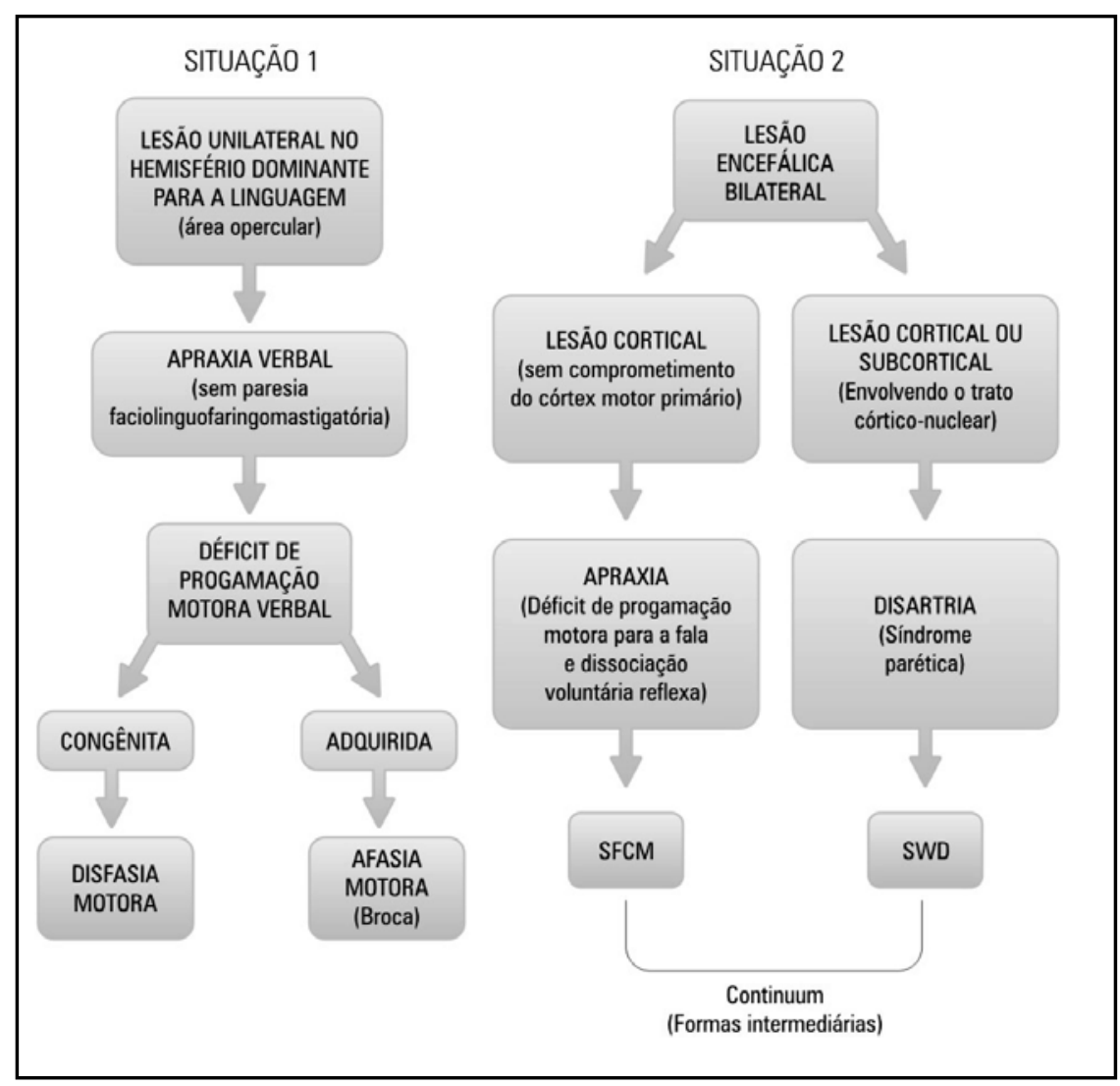

Fig 3. Fluxograma para diferenciação das síndrome de Worster-Drought (SWD) e síndrome de Foix-Chavany-Marie (SFCM).

a síndrome de Worster-Drought e a síndrome perisylviana. Clark et al. ${ }^{2}$ também registraram casos de paralisia pseudobulbar associada a apraxia oculomotora por lesão nas vias motoras descendentes na porção anterior da cápsula interna. $O$ envolvimento do VII nervo craniano, a tetraplegia, os problemas cognitivos, epilepsia e anormalidades congênitas reforçam o caráter pouco específico da lesão que envolveria áreas mais difusas, provavelmente por defeito em fase precoce do desenvolvimento fetal. Kuzniecky et al. foram os primeiros a encontrar similaridades nos pacientes com os dois tipos de síndrome, iniciando o pensamento, defendido por Christen et al. ${ }^{11} \mathrm{em}$ renomear a síndrome opercular ou de Foix-Chavany-Marie em síndrome de Worster-Drought.

Os achados radiológicos da síndrome de WosterDrought e da síndrome de Foix-Chavany-Marie são singulares. Na primeira, à tomografia computadorizada, podem ser encontradas atrofia do hemisfério cerebral direito, hipodensidade periventricular esquerda e na região têmporo-parietal; a ressonância magnética evidencia polimicrogiria perisylviana bilateral, heterotopia de substancia cinzenta subependimária, agenesia parcial do corpo caloso, atrofia cerebral moderada, atrofia cerebelar moderada com pobre diferenciação entre substância branca e cinzenta, aumento do sinal na cápsula interna em T2, assimetria cerebelar e tamanho reduzido da ponte ${ }^{1}$. Por outro lado, na FCMS existe uma atrofia cortical assimétrica, mais intensa à esquerda e predominando na região frontal inferior, notavelmente na região opercular, evidenciada na ressonância magnética. PET-scan e SPECT confirmam uma diminuição esperada no fluxo sanguíneo e metabolismo cerebral, mais proeminente no giro frontal inferior esquerdo e no córtex pré-motor, estendendo-se bilateralmente nos casos avançados ${ }^{1}$. No caso em questão, a ressonância magnética do encéfalo revelou alterações mais sugestivas da síndrome de Worster-Drought.

Acreditamos que, de acordo com as descrições originais dos autores que deram nome às síndromes, podemos considerá-las como situações clinicamente distintas e recomendamos a utilização do fluxograma (Fig 3) para melhor condução diagnós- 
tica. No caso apresentado, a interpretação dos resultados da avaliação psicológica sugere um desempenho abaixo da maturidade esperada para sua idade. Apesar das limitações nas respostas, verificou-se que através de atividades de acompanhamento individualizado seu desenvolvimento pode ser otimizado.

Considerando-se que uma das hipóteses sobre a etiologia da síndrome de Worster-Drought é a ocorrência de agenesia ou disgenesia do trato córtico-nuclear, questionamos a possibilidade de participação de drogas teratogênicas, como o misoprostol, em sua patogênese, como já foi identificado em pacientes com síndrome de Moebius, na qual existe agenesia de núcleos de nervos crania$\operatorname{nos}^{12}$. Pressupõe-se que o uso de teratógenos interfere nos primórdios da vida intra-uterina por interferir na circulação uterina fetal, provocando a referida patologia (teoria teratogênica) ${ }^{13}$. Nos casos mais graves da SWD, o melhor prognóstico fonoaudiológico é alcançado pelo desenvolvimento de estratégias alternativas, como suplemento na comunicação destas crianças, enfatizando a grande ajuda oferecida pelos gestos, expressão facial, símbolos, escrita e até sintetizador de fala. A taxa de progresso na terapia fonoaudiológica dependerá da dificuldade motora, da motivação para se comunicar e das dificuldades de aprendizado associadas a cada caso. No caso apresentado, considerando a idade da paciente e a resposta insatisfatória às intervenções fonoterápica e miofuncional prévias, optamos por inseri-la em programa de comunicação alternativa.

Em relação à sialorréia observada, a conduta adotada foi a de orientação à família em relação a exercícios miofuncionais específicos. WorsterDrought ${ }^{1}$ relatou que as cirurgias de palatoplastia ou palato-faringoplastia podem melhorar o problema em alguns casos. Estes procedimentos promovem o fechamento do esfíncter oro-nasal, o que aumenta o poder de controle da saliva na cavidade oral, facilitando a deglutição.

A etiologia da síndrome de Worster - Drought é mal conhecida e ela é subdiagnosticada. Questionamos se o uso de drogas abortivas poderia de- sempenhar papel importante na gênese da síndrome. No entanto, é necessário realizar estudos analíticos para identificar uma relação de causa-efeito. Intervenção fonoaudiológica nestes casos é de fundamental importância, não somente para melhor orientação em relação à alimentação, mas principalmente em relação ao desenvolvimento da linguagem. Em relação aos aspectos cognitivos, outros estudos devem ser realizados com o objetivo de procurar parâmetros para compreensão do desenvolvimento da aprendizagem e para o fornecimento de subsídios que fundamentem a intervenção psicopedagógica em crianças portadoras de Worster-Drought.

Na literatura brasileira não foi encontrado nenhum estudo sobre a síndrome, sendo a condição, portanto, subdiagnosticada. Faz-se necessário o desenvolvimento de pesquisas que caracterizem a incidência desta entidade na nossa população.

\section{REFERÊNCIAS}

1. Worster-Drought C. Suprabulbal paresis: congenital suprabulbar paresis and its differential diagnosis, with special reference to acquired suprabulbal paresis. Dev Med Child Neurol 1974;16:1-33.

2. Clark M, Carr L, Reilly S, Neville B. Worster-Drought syndrome, a mild tetraplegic perisylvian cerebral palsy: review of 47 cases. Brain 2000;123:2160-2170.

3. Maw-Yuan A, On-Kee L, Yen-Lin W. Foix-Chavany-Marie syndrome. Chin Med J 2001;64:540-544.

4. Nevo Y, Segev Y, Gelman Y, Rieder-Grosswasser I, Harel S. WorsterDrought and congenital perisylvian syndromes: a continuum? Ped Neurol 2001;24:153-155.

5. Weiss, MLL. Psicopedagogia clínica: uma visão diagnóstica dos problemas de aprendizagem escolar. Rio de Janeiro: DP\&A, 2002.

6. Calwson A. Bender Infantil: manual de diagnóstico clínico. Porto Alegre: Artes Médicas, 1992.

7. Zorzi JL. Diferenciando alterações da fala e da linguagem. In Marchesan IQ (ed). Fundamentos em fonoaudiologia - aspectos clínicos da motricidade oral. Rio de Janeiro: Guanabara Koogan, 1998:59-74.

8. Weller M. Anterior opercular cortex lesions cause dissociated lower crabial nerve palsies and anarthria but no aphasia: Foix-ChavanyMarie syndrome and autonomic voluntary dossociation revisited. J Neurol 1993;240:199-208.

9. Ortiz KZ. Avaliação e terapia dos distúrbios neurológicos da linguagem e fala. In Filho OL (ed). Tratado de fonoaudiologia. São Paulo: Roca, 1997:939-958.

10. Murdoch BE. Desenvolvimento da fala e distúrbios da linguagem: uma abordagem neuroanatômica e neurofisiológica. Revinter, 1997.

11. Christen HJ, Hanefeld F, Kruse E, Imhauser S, Ernst JP, Finkenstaedt M. Foix-Chavany-Marie (anterior operculum) syndrome in childhood: a reapprasail of Worster-Drought syndrome. Dev Med Child Neurolol 2000;42:22-132.

12. Reed H, Grant W. Möebius syndrome. Br. Bone Joint Surg 1953;35:437-44.

13. Lipson AH, Webster WS, Brown-Woodman PDC, Osborn RA. Reply to comments on Möebius syndrome: animal model - human: correlation and evidence for a brainsteam vascular etiology. Teratology 1991;43:557. 